\title{
BMJ Open International Mind, Activities and Urban Places (iMAP) study: methods of a cohort study on environmental and lifestyle influences on brain and cognitive health
}

\author{
Ester Cerin (1) , ${ }^{1,2}$ Anthony Barnett, ${ }^{1}$ Basile Chaix, ${ }^{3}$ Mark J Nieuwenhuijsen, ${ }^{4}$ \\ Karen Caeyenberghs, ${ }^{5}$ Bin Jalaludin, ${ }^{6}$ Takemi Sugiyama, ${ }^{1,7}$ James F Sallis, ${ }^{1,8}$ \\ Nicola T Lautenschlager, ${ }^{9}$ Michael Y Ni, ${ }^{2}$ Govinda Poudel, ${ }^{1}$ \\ David Donaire-Gonzalez, ${ }^{1}$ Rachel Tham, ${ }^{1}$ Amanda J Wheeler, ${ }^{1}$ Luke Knibbs, ${ }^{10}$ \\ Linwei Tian, ${ }^{2}$ Yih-kai Chan, ${ }^{1}$ David W Dunstan, ${ }^{11}$ Alison Carver, ${ }^{1}$ Kaarin J Anstey ${ }^{12,13}$
}

To cite: Cerin E, Barnett A, Chaix B, et al. International Mind, Activities and Urban Places (iMAP) study: methods of a cohort study on environmental and lifestyle influences on brain and cognitive health. BMJ Open 2020;10:e036607. doi:10.1136/ bmjopen-2019-036607

- Prepublication history for this paper is available online To view these files, please visit the journal online (http://dx.doi org/10.1136/bmjopen-2019036607).

Received 21 December 2019 Revised 11 February 2020 Accepted 19 February 2020

Check for updates

(C) Author(s) (or their employer(s)) 2020. Re-use permitted under CC BY-NC. No commercial re-use. See rights and permissions. Published by BMJ.

For numbered affiliations see end of article.

Correspondence to

Professor Ester Cerin;

Ester.Cerin@acu.edu.au

\section{ABSTRACT}

Introduction Numerous studies have found associations between characteristics of urban environments and risk factors for dementia and cognitive decline, such as physical inactivity and obesity. However, the contribution of urban environments to brain and cognitive health has been seldom examined directly. This cohort study investigates the extent to which and how a wide range of characteristics of urban environments influence brain and cognitive health via lifestyle behaviours in mid-aged and older adults in three cities across three continents.

Methods and analysis Participants aged $50-79$ years and living in preselected areas stratified by walkability, air pollution and socioeconomic status are being recruited in Melbourne (Australia), Barcelona (Spain) and Hong Kong (China) ( $n=1800$ total; 600 per site). Two assessments taken 24 months apart will capture changes in brain and cognitive health. Cognitive function is gauged with a battery of eight standardised tests. Brain health is assessed using MRI scans in a subset of participants. Information on participants' visited locations is collected via an interactive web-based mapping application and smartphone geolocation data. Environmental characteristics of visited locations, including the built and natural environments and their by-products (e.g., air pollution), are assessed using geographical information systems, online environmental audits and self-reports. Data on travel and lifestyle behaviours (e.g., physical and social activities) and participants' characteristics (e.g., sociodemographics) are collected using objective and/or self-report measures.

Ethics and dissemination The study has been approved by the Human Research Ethics Committee of the Australian Catholic University, the Institutional Review Board of the University of Hong Kong and the Parc de Salut Mar Clinical Research Ethics Committee of the Government of Catalonia. Results will be communicated through standard scientific channels. Methods will be made freely available via a study-dedicated website.

Trial registration number ACTRN12619000817145.
Strengths and limitations of this study

- By recruiting participants from residential areas stratified by walkability, air pollution and socioeconomic status from three cities varying in exposures and lifestyle behaviours, this study will allow a robust estimation of dose-response relationships of urban design and the relevant by-products (e.g., pollution) with brain and cognitive health.

- This study will provide a comprehensive assessment of multiple environmental factors and multiple lifestyle behaviours by which environments may affect brain and cognitive health. This will allow improved understanding of which environmental characteristics are related to brain and cognitive outcomes and by which mechanisms.

- The focus on locations visited for daily activities rather than only residential neighbourhoods will enable a more accurate estimation of individual exposure to features of the urban environment.

- The comprehensive assessment of lifestyle behaviours and individual-level moderators (apolipoprotein E genotype, physical health and personality traits) will assist the identification of optimal patterns of lifestyle activities that can be integrated in individually tailored lifestyle interventions, taking into account individual genetic factors and environmental living conditions.

- Current resources can support only two assessments, 24 months apart. This may not be a sufficiently long period to detect changes in certain exposures and/or outcomes.

\section{INTRODUCTION}

Dementia prevention is a global health priority. Around 40-50 million people suffer from dementia worldwide and these figures are expected to almost double by $2030^{12}$ due to population ageing. ${ }^{3}$ Mild cognitive impairment (MCI) that is not severe enough to meet 
a diagnosis of dementia also causes significant disability and healthcare costs. ${ }^{4}$ The prevalence of MCI across eight countries, determined using various criteria, ranges from $2 \%$ to $21 \% .^{5}$ As no effective pharmacological treatment for dementia and MCI is yet available, prevention is the best strategy to lower their incidence, associated disability and costs.

An important cornerstone of a global strategy for longterm dementia and MCI prevention is cognitive health promotion during adulthood. Cognitive health promotion aims to minimise cognitive decline among those with MCI, and to maintain cognitive function in healthy individuals by targeting relevant modifiable risk factors that reduce the risk of neurodegeneration and increase cognitive resilience, ${ }^{67}$ a concept used to explain a person's capacity to maintain normal cognitive function in the presence of brain pathology.

A report by the Institute of Medicine (USA) on cognitive ageing and the maintenance of cognitive function identified solid or moderate evidence for several modifiable factors that contribute to cognitive health in midlife and late life, including: physical activity; intellectual activities; social activities; air pollution; reduction or management of cardiovascular disease risk factors (e.g., smoking, hypertension and diabetes) and adequate sleep. ${ }^{8}$ Importantly, it was noted that much less was known about lifestyle risk factors for cognitive health across the lifespan compared with dementia-based outcomes. The report also highlighted the importance of finding effective approaches to sustaining behavioural changes that promote healthy cognition across the lifespan. ${ }^{8}$

Environmental and policy interventions targeting cognitive health-enhancing behaviours and other exposures (e.g., air pollution) are particularly relevant in this context because they can influence entire populations for a sustained amount of time. ${ }^{9}$ In line with these ideas, the Global Observatory for Ageing and Dementia Care highlighted the need for evidence-based, multilevel, multisectoral (urban planning and design, transportation, residential aged care, health) strategies to yield significant, large-scale, sustainable reductions in incidence of dementia. ${ }^{1}$ However, empirical evidence to inform multisectoral strategies to reduce dementia risk and promote cognitive health is lacking because most research in these fields has focused on individual-level factors (e.g., lifestyle behaviours and biomarkers), while broader environmental factors (e.g., neighbourhood attributes) and people's interactions with the environment have been largely overlooked.

As over half of the world's population lives in cities and the rate of urbanisation is rapidly increasing, ${ }^{10}$ it is particularly important to study the effects of urban environments and their characteristics on cognitive health. Urbanisation leads to economic development and better access to education, culture and healthcare, ${ }^{11}$ which are deemed to benefit physical and cognitive health. However, rapid, and often unplanned, urban growth and densification is typically associated with poverty, crime, air pollution, noise, environmental degradation and population demands that outstrip service capacity. These negative conditions have adverse effects on human health and the health of the planet. ${ }^{11}$ Evidence-based, healthoriented urban design is increasingly recognised as a key strategy to improve health outcomes through the modification of the physical features of urban environments, ${ }^{12}$ as documented in a recent series of papers published in The Lancet. ${ }^{13}$ However, such a strategy requires solid knowledge of the health impacts of the key aspects of urban environments.

A substantial number of studies have reported significant effects of aspects of the urban environment (residential neighbourhoods, in particular) on physical activity, cardiovascular health and obesity, ${ }^{14-18}$ which are established determinants of cognitive health. ${ }^{8}$ However, the role of urban environments in relation to brain and cognitive health has received little attention. Preliminary support for the importance of urban design for brain and cognitive health comes from a few recent studies, which found mixed land use, access to various types of destinations and/or access to public transport to be predictive of better cognitive health ${ }^{19}{ }^{20}$ and brain health. ${ }^{21}$ Yet, the findings have not been consistent. Unexpected negative or curvilinear associations between measures of destination accessibility and cognitive function have also been reported. ${ }^{22} 23$ These contrasting findings may be due to unadjusted confounders, such as exposure to air and noise pollution or green spaces. ${ }^{24}$

Using a broad socioecological framework, ${ }^{9}$ we hypothesise that urban environments affect brain and cognitive health indirectly by influencing travel (e.g., mode of transport and visited locations) and other lifestyle behaviours (e.g., engagement in social, intellectual and physical activities) and, more directly, by exposing residents to potentially harmful (e.g., noise and pollution) environmental factors (urban design by-products in figure 1). For example, high-density neighbourhoods with easy access to shops, services and parks are important enablers of active travel and recreational physical activity, ${ }^{146}$ which, in turn, benefit brain and cognitive health by enhancing affective states, cardiometabolic health, neurogenesis, brain connectivity and by reducing inflammation and oxidative stress. ${ }^{1825}$ However, all these positive effects may vanish if, as a result of being physically active in a high-density neighbourhood, individuals become exposed to harmful environmental factors, such as traffic-related air pollution and noise, which damage brain and cognitive health by increasing neuroinflammation and oxidative stress. ${ }^{24} 2627$ To understand the full impact of urban environments on brain and cognitive health, it is important to quantify the joint and independent contributions of all key aspects of urban design and related environmental exposures (figure 1). This information is currently unavailable due to the dearth of studies in this area. ${ }^{19} 24$

The evidence that participation in physical activity benefits brain and cognitive health is relatively strong, while that related to dietary intake, intellectual, social 


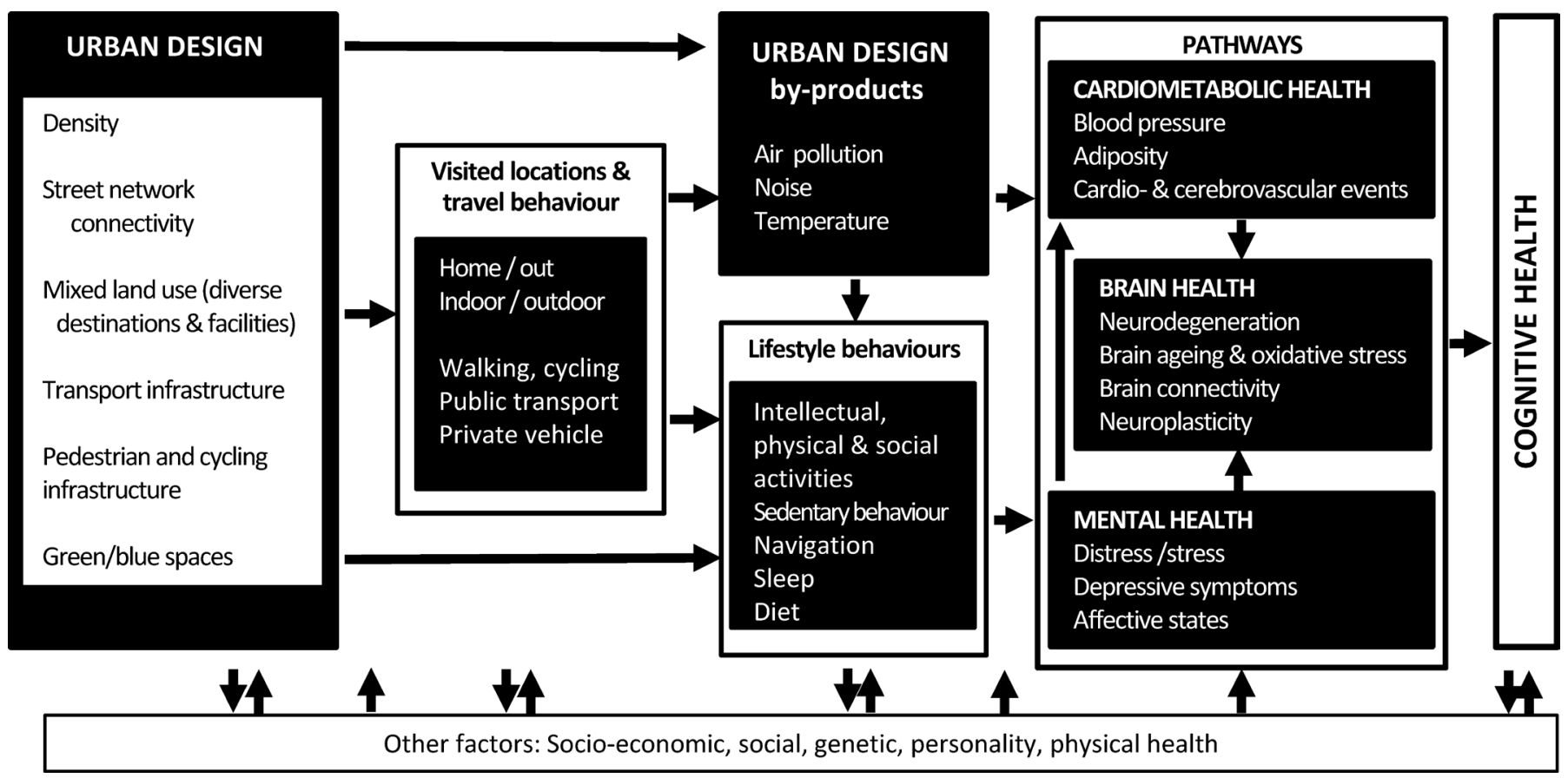

Figure 1 Conceptual ecological model of the effects of urban design on cognitive health examined in the iMAP study. iMAP, International Mind, Activities and Urban Places.

and navigational (wayfinding) activities, sedentary behaviour and sleep is insufficient or less compelling. ${ }^{1826}$ While there is strong evidence that all six aspects of urban design depicted in figure 1 promote active transport and physical activity, especially in older adults, ${ }^{14} 15$ the extent to which they affect other lifestyle behaviours and the choice of visited locations is not known. The latter issue has important health implications as, in conjunction with the chosen mode of transport, it determines the level of exposure to negative by-products of urban design and, hence, their effects on biomarkers and cognitive health. Considering that living near a major road has recently been identified as a potentially major risk factor for dementia, ${ }^{27} 29 \%$ of strokes have been attributed to air pollution, ${ }^{28}$ and the number of motorised vehicles in many countries continues to rise, ${ }^{29}$ it is crucial to accurately quantify the direct and behaviour-mediated impacts of traffic-related air and noise pollution on brain and cognitive health by identifying where people's activities take place.

In addition to lifestyle behaviours, person-level characteristics, such as genetic factors ${ }^{8}$ personality traits and chronic diseases, ${ }^{8}$ are key determinants of cognitive health. For example, the apolipoprotein E $(A p o E) \varepsilon 4$ and $\varepsilon 2$ alleles, brain injury and the personality traits of neuroticism and conscientiousness have all been associated with levels and/or biological mechanisms of cognitive decline. ${ }^{122}$ Importantly, these personal factors are also potential moderators of the effects of the urban environments on cognitive health. Studies have reported $A p o E$ genotype moderation effects on associations of cognitive function with cognition-promoting activities ${ }^{30}$ and environmental stressors ${ }^{31}$ including air pollution. ${ }^{32}$ Genetic factors and personality traits may also determine how individuals interact with the environment and the biological pathways via which the environment affects cognition. For example, the negative effect of neuroticism on cognitive health can be attributed to elevated autonomic reactivity ${ }^{33} 34$ and consequent chronic elevations in cortisol (a marker of stress), which has been linked to atrophy in most regions of the cerebral cortex. ${ }^{35}$ Thus, exposure to environmental stressors (e.g., noise, signs of crime) and other challenges (e.g., crowding and attentional overload present in high-density areas) may be (more strongly) associated with worse cognitive health in those with higher levels of neuroticism. Given the above and in line with socioecological models of health, ${ }^{9}$ it is important to adopt an interactionist multilevel approach to the study of the impacts of urban environments on brain and cognitive health, something that has not been done much to date.

The International Mind, Activities and Urban Places (iMAP) project is a collaborative multicity prospective cohort study designed to address the limitations of current research. Its overarching objective is to examine the extent to which, how and for whom a wide range of characteristics of urban environments influence brain and cognitive health in mid-aged and older community dwellers. The specific aims of the project are to: (1) identify aspects of urban environments that are potential determinants of brain and cognitive health; (2) investigate the extent to which environmental influences on brain and cognitive health are explained by travel behaviour, lifestyle activities (physical, social, intellectual and navigational activities, sedentary behaviour and sleep) and exposure to environmental hazards (e.g., air 
and noise pollution); (3) identify brain imaging measures and markers of cardiometabolic health mediating the effects of the urban environment and lifestyle activities on cognitive health and (4) examine the moderating effects of genetics, personality and baseline chronic diseases on the relationships of characteristics of the urban environment with cognitive health and their mediating pathways. iMAP is the first cohort study designed to examine all these issues simultaneously and the first to employ a common methodology across cities with diverse urban environments to explore environmental determinants of brain and cognitive health in mid-aged and older adults. This paper provides a general overview of the research methods of iMAP.

\section{METHODS AND ANALYSIS}

The standardised checklist based on the Strengthening the Reporting of Observational Studies in Epidemiology recommendations was used to ensure that all relevant aspects of study design and data collection were addressed. ${ }^{36}$

\section{Design}

iMAP is a multisite observational longitudinal study on environmental and lifestyle determinants of brain and cognitive health in 1800 adults aged 50-79 years at baseline. This cohort study is currently being conducted in three locations: Melbourne (Australia), Barcelona (Spain) and Hong Kong (China). As shown in table 1, these cities vary substantially in several key environmental exposures (population density, street intersection density, land use mix, air pollution) and lifestyle behaviours (physical activity) of interest, as well as in the prevalence estimates of dementia (although unstandardised by age and assessment criteria). By conducting the study in three diverse cities, we maximise the variability in environmental exposures which may allow an accurate estimation of the dose-response relationships between aspects of the urban environment, lifestyle behaviours and cognitive health. ${ }^{37}{ }^{38}$ iMAP will also make it possible to examine if the effects of the environment on cognitive health vary by geographical locations and cultures. This information is important to guide globally and locally relevant urban planning and transportation policies.

Each study site aims to recruit 600 participants from preselected administrative units (neighbourhoods) stratified by walkability, air pollution and socioeconomic status (SES). Data on sociodemographic characteristics, lifestyle activities and their locations, transport behaviour, health status, neighbourhood attributes, social networks, affective states, personality traits, $A p o E$ genotype and cognitive function will be collected in the full sample. Subsamples of participants will be invited to undertake MRI brain scans. Data collection will be conducted at two time points 24 months apart. Subsequent waves of data collection and additional study sites will be dependent on funding.

\section{Neighbourhood and participant selection Neighbourhood selection}

This study uses a stratified two-stage sampling design whereby administrative units varying in SES, walkability and air pollution are first selected and participants are then recruited within the preselected administrative units. ${ }^{39}$ Administrative units in each study site are represented by the smallest administrative areas with publicly available census data: Statistical Areas 1 in Melbourne, Tertiary Planning Units in Hong Kong and Census Areas in Barcelona. This recruitment strategy maximises the variability of environmental exposures within each site, helps to address collinearity issues, avoids reciprocal confounding among these broad environmental dimensions and, thus, assists the accurate estimation of

\begin{tabular}{llll}
\hline Table 1 & Environmental attributes, lifestyle behaviours and dementia prevalence in & Melbourne, Barcelona and Hong Kong \\
\hline Characteristics & Melbourne (Australia) & Barcelona (Spain) & Hong Kong (China) \\
\hline Population density in urban areas $\left(\text { people } / \mathrm{km}^{2}\right)^{\star}$ & 3200 & 16000 & 25900 \\
Street intersection density (intersections $\left./ \mathrm{km}^{2}\right) \dagger$ & 70 & 223 & 55 \\
Land use mix (entropy index; range: $0-1) \ddagger$ & 0.15 & 0.19 & 0.50 \\
Mean annual concentration of $\mathrm{PM}_{2.5}\left(\mu \mathrm{g} / \mathrm{m}^{3}\right)$ & 8 & 14 & 63 \\
Mean annual concentration of nitrogen dioxide $\left(\mu \mathrm{g} / \mathrm{m}^{3}\right)$ & 16 & 50 & 95 \\
Range in average monthly temperature $\left({ }^{\circ} \mathrm{C}\right)$ & $11-22$ & $10-25$ & $17-30$ \\
Prevalence of health-enhancing physical activity§ & $55 \%{ }^{98}$ & $77 \%{ }^{99}$ & $85 \%{ }^{100}$ \\
Prevalence of dementia & $9.0 \%{ }^{3} \boldsymbol{q}$ & $2.4 \%{ }^{101}$ ** & $3.3 \%{ }^{102}$
\end{tabular}

${ }^{*}$ Based on city-specific census data.

†Computed using city-specific street network data.

fLand use mix entropy index ${ }^{40}$ based on city-specific data on five land use categories: residential, commercial, civic/institutional/educational; industrial; recreational and park.

§Percentage adults (18+ years) accumulating 150+ min week of physical activity as measured by the International Physical Activity

Questionnaire.

ףIn 65+ year-olds.

${ }^{* *}$ In $70+$ year-olds. $\mathrm{PM}_{2.5}=$ particulate matter with aerodynamic diameter $<2.5 \mu \mathrm{m}$. 
dose-response relationships of environmental characteristics with mediators and outcomes. The walkability index consists of indicators of street connectivity, residential density and land use mix. ${ }^{39}$ The latter indicator is represented by an entropy index ranging from 0 to $1^{40}$ based on data from five land use categories (residential, commercial, industrial, educational/civic/institutional and park/ recreational). The air pollution index is based on mean annual concentrations of nitrogen dioxide $\left(\mathrm{NO}_{2}\right)$, most of which is from motor vehicle emissions in urban areas. ${ }^{41}$ Estimates of $\mathrm{NO}_{2}$ concentration are derived from land use regression models. ${ }^{42}$ Administrative units are first ranked by walkability and those in the top and bottom 4 or 5 deciles (depending on the within-site distribution and range of values) are selected and classified as high and low walkable. The same method is then used to classify each of the two walkability strata into high and low pollution. Finally, administrative units belonging to the resulting four walkability by pollution strata are ranked by SES (median household income or equivalent SES censusbased indicator) and the top and bottom 4 or 5 deciles classified as high and low SES. Approximately 15 administrative units from within each of the eight walkability by pollution by SES strata (a total of $\sim 120$ administrative units per study site) are selected based on their censusbased number of residents aged $50-79$ years $(n>50)$ and level of representativeness of their respective administrative unit stratum quantified using the following formula:

$$
\begin{aligned}
& \text { Representativeness } \text { score }_{a u}=w_{1 . s} * \text { Walkability z } \text { score }_{a u}+ \\
& \frac{1}{2}\left(w_{2 . s} * \text { Air pollution z } \text { score }_{a u}\right)+\frac{1}{3}\left(w_{3 . s} * \text { SES z } \text { score }_{a u}\right)
\end{aligned}
$$

where $a u$ refers to a specific administrative unit within a study site, $w_{1 \ldots . .3}$ are weights $(1$ or -1$)$ given to a specific administrative unit stratum (e.g., high walkable, low air pollution, low SES) with 1 denoting a high level and -1 denoting a low level of a specific environmental characteristic. Walkability, air pollution and SES are, respectively, assigned weights of $1,1 / 2$ and $1 / 3$ for administrative unit selection purposes to reflect their pertinence for the present study. The built and natural environments are the main focus of this study, while air pollution is considered a by-product of the built and natural environments. SES is treated as a potential confounder partially accounted for by the stratified sampling strategy. To minimise spatial clustering effects on the effective sampling size, ${ }^{43}$ in selecting administrative units, clusters of more than two adjacent administrative units are avoided.

\section{Participant selection and recruitment}

Participants are being recruited over 2 years equally from all administrative unit (neighbourhood) types to control for confounding effects of seasonal variations in weather conditions on the associations between environment, lifestyle behaviours and cognitive function and ensure generalisability. ${ }^{44}$

After raising community awareness about the study through local newspapers and flyer/poster dissemination, participants aged 50-79 years and residing independently in the selected administrative units are sampled using the most appropriate strategy for a specific study site in terms of feasibility and sample representativeness. We aim to recruit a minimum of three and a maximum of eight participants balanced by sex and age (age strata: 50-59 years; 60-69 years and 70-79 years) from each selected administrative unit. While the preferred strategy would have been stratified random sampling from a comprehensive sampling frame (e.g., electoral roll), due to current data protection laws, other sampling methods need to be implemented.

Melbourne (Australia) is employing door-to-door recruitment involving three flyer drops and two doorknocks on different days and times of the week. In administrative units with high-rise residential buildings where door-knocking is not permitted, this strategy is complemented with an 8-hour street intercepts and additional flyer mail-outs. This recruitment protocol is applied to all streets of the administrative unit. In administrative units with fewer than five potentially eligible participants recruited via door-knocking and with a low number of contacted households $(>60 \%$ households with no one at home), door-knocking is complemented with recruitment from community organisations (e.g., community centres, recreational centres, churches).

Barcelona (Spain) employs street intercepts as the main recruitment strategy due to the inability to access households in multistorey residential buildings, which are highly prevalent. In administrative units with fewer than five potentially eligible participants recruited via street intercepts, potential participants are also contacted via primary care physicians and health centres located in the administrative unit.

Hong Kong (China) uses a large population-based cohort - the FAMILY Cohort ${ }^{45}$ - as the sampling frame for the iMAP study. The FAMILY Cohort is a Hong Kong territory-wide longitudinal study of health, happiness and family harmony which was established in 2009-2011 with 46001 participants ( $25 \%$ aged 60 or above) at baseline and a retention rate of $\sim 70 \%$ at follow-up (2011-2014). Potential participants for the iMAP study are randomly selected within sex and age group strata from a pool of FAMILY Cohort participants who reside in the $\sim 120$ preselected administrative units. In all study sites, if recruitment in a specific administrative unit results in a pool of more than 5-8 potentially eligible participants, random selection stratified by sex and age group is employed to choose 5-8 participants from the pool.

Potentially eligible participants selected for the study are contacted by telephone for preliminary eligibility screening purposes, sent a study information sheet and a consent form, and booked in for a detailed face-to-face eligibility screening and initial baseline assessment. Inclusion criteria are: (1) being 50-79 years of age; (2) living independently (i.e, not in congregate or assisted living housing) in one of the selected administrative units; (3) having lived at the current address for $\geq 12$ months; (4) being able to speak and write in English (Melbourne 
arm), Spanish or Catalan (Barcelona arm) or Chinese (Hong Kong arm); (5) reporting to be able to walk for more than 5 min without significant difficulty. Exclusion criteria are: (1) existing diagnosis of dementia and/or major depressive disorder (assessed via phone screening interview) or probable dementia and/or clinical depression (assessed via face-to-face screening); (2) presence of major illnesses/neurological conditions that would prevent assessment completion. ${ }^{46}$ During the face-toface eligibility screening, probable dementia and clinical depression are determined using the Montreal Cognitive Assessment (MoCA) ( $\geq 24$ points $)^{47}$ and the Patient Health Questionnaire-9 (<10 points) ${ }^{48}$ respectively.

\section{Sample size}

iMAP aims to recruit 600 participants per site, resulting in 1800 participants across the three sites. The sizes of the total (mediated and unmediated) effects of single environmental attributes on levels/changes in behaviours/ health outcomes are likely to be $\sim 1 \%-2 \%$ of explained outcome variance for each environmental factor, while the sizes of the direct effects of lifestyle behaviours may be $\sim 10 \%-20 \%$ of outcome variance. ${ }^{30} 38$ In a regression model with $>20$ covariates and continuous outcomes (scores on cognitive function tests), 518 participants would be needed to detect the expected effect size $(1.5 \%$ of variance) with $80 \%$ power and a two-tailed probability level of $5 \%$, assuming uncorrelated residuals. As participants are recruited from $\sim 120$ preselected administrative units (representing clusters) in each site, data from the same administrative units will likely be correlated. The magnitude of clustering in lifestyle behaviours and brain/ cognitive health outcomes estimated by the residual intraclass correlation, which, in previous studies adopting a similar sampling strategy, has been on average $\sim 0.005 .^{38}$ Considering a clustering effect of this magnitude, 533 participants per study site would allow the detection of the expected effect size ( $1.5 \%$ of variance) with $80 \%$ power. Thus, the proposed sample size of 600 participants per site would provide sufficient statistical power for baseline, cross-sectional, site-specific regression analyses as well as pooled analyses. Conservatively assuming a 20\% drop-out rate at the second assessment ( 2 years postbaseline) ${ }^{49}$ the final sample size will be 426 per site and 1280 across the three sites. These samples sizes will, respectively, achieve $80 \%$ power to detect effect sizes equivalent to $1.9 \%$ and $0.6 \%$ of variance explained in longitudinal analyses. With regard to MRI brain imaging outcomes, our study indicated that environmental attributes may explain $\sim 5 \%$ of the variance in MRI brain volumetric measures, ${ }^{21}$ requiring 152 participants to achieve $80 \%$ power in site-specific cross-sectional regression analyses. With a conservative $20 \%$ drop-out rate ${ }^{49}$ and 200 participants at baseline, the final sample size of 160 participants per site and 560 in total will, respectively, be able to detect effect sizes equivalent to $5.0 \%$ and $1.4 \%$ of variance explained in longitudinal analyses.

\section{Exposures, outcomes and covariates}

\section{Exposures}

Environmental exposures are assessed using objective and self-report measures. Objective assessment of the participants' environments entails linking geocoded participants' visited locations and connecting routes to objective environmental data using geographical information system (GIS) and the online version of the Microscale Audit of Pedestrian Streetscapes (MAPS) Global. $^{50}{ }^{51}$ Self-reported habitual, frequently visited activity locations (e.g., home, workplace, places for recreational, educational, volunteering activities and shopping) are captured and geocoded through a version of the Vizualization and Evaluation of Route Itineraries, Travel destinations, and Activity Spaces (VERITAS) interactive map-based questionnaire ${ }^{52}$ developed for iMAP, while a geolocation application for smartphones is used to collect objective data on participants' visited locations and connecting routes during a 7-day monitoring period. For each participant, street network and/or Euclidean (i.e., 'as the crow flies') buffers of different sizes are created around geocoded habitual locations (e.g., $0.5 \mathrm{~km}, 1 \mathrm{~km}$ radii buffers) and smartphone spatial tracks (e.g., $0.1 \mathrm{~km}$, $0.2 \mathrm{~km}$ radii buffers). The type and size of the buffers used depend on the environmental attribute and outcome studied. For example, small $100 \mathrm{~m}$ radius crow-fly buffers along connecting routes may be used to characterise exposure to air pollution ${ }^{53}$ and define attributes within eyesight range, while $1 \mathrm{~km}$ radius street network buffers surrounding reference locations (i.e., locations around which individuals organise their daily activities and are relatively obligated to go to $)^{54}$ are employed to quantify land use mix as a predictor of utilitarian walking. ${ }^{55}$

GIS data are linked to the buffers to create participantspecific objective measures of the environment (urban design and urban design by-products in figure 1). Spatial data temporally aligned with the cohorts' data collection years are sought. Where spatial data are not available at the same time period, the closest available time period prior to participants' assessments is used. Urban design constructs that are being assessed using GIS encompass dwelling density, street network connectivity, availability and spatial accessibility of destinations and green/blue spaces, and transport, pedestrian and cycling infrastructures (figure 1 and table 2). Two aspects of pedestrian infrastructure-namely, pavements and traffic safetyare being assessed using online MAPS Global. ${ }^{50}{ }^{51}$ Using images from Google Earth StreetView, trained auditors are using MAPS Global to collect information on traffic safety and pavement quality along the routes that connect residential/workplace addresses to the nearest commercial areas. VERITAS iMAP is used to assess residential building characteristics (type of housing, availability of recreational areas, natural sights).

Urban design by-products are quantified using GIS methods. Air pollutants of interest are fine particulate matter with an aerodynamic diameter $<2.5 \mu \mathrm{m}\left(\mathrm{PM}_{2.5}\right)$ and $\mathrm{NO}_{2}$ because there is evidence that they may be risk factors 
Table 2 Correlates of brain and cognitive health and covariates, moderators and mediators of associations between environmental attributes and brain and cognitive health in the iMAP study by data collection component

\section{Data collection Constructs measured (type component of assessment)}

Initial face-to-face Blood pressure (O) assessment

Anthropometric measures (O)

Depressive symptoms (S)

ApoE genotype (O)

Physical function (O)

Health status (S)

\section{Construct in conceptual}

model (figure 1)

(role in model)

Cardiometabolic health: blood Blood pressure assessed using a blood pressure pressure (ME)

Cardiometabolic health: adiposity (ME)

Mental health: depressive symptoms (ME)

Other factors: genetic (C, MO) DNA collected at baseline using a commercial kit for saliva collection. Genotype determination performed at a local lab.

$\begin{array}{ll}\text { Physical function }(\mathrm{O}) & \begin{array}{l}\text { Other factors: physical health } \\ (\mathrm{C}, \mathrm{MO})\end{array} \\ \text { Health status }(\mathrm{S}) & \begin{array}{l}\text { Cardiometabolic health: } \\ \text { cardiovascular and } \\ \text { cerebrovascular events (ME) } \\ \text { Other factors: physical health } \\ (\mathrm{C}, \mathrm{MO})\end{array}\end{array}$

Self-administered Socioeconomic, demographic survey (S) Sleep (S)

Other factors: socioeconomic (C, MO)

Lifestyle behaviour: sleep (ME)

Dietary patterns (S)
Neuroticism and
conscientiousness (S) Lifestyle behaviours: $\operatorname{diet}(\mathrm{C})$ Neighbourhood self-selection NA (C) (S)

(S)

7 day field assessment

Visited locations and travel behaviours (\# trips, duration, routes and modes of transport) (TD, O)

Physical activity (frequency, amount, intensity) (O)
Lifestyle behaviours: physical activity (ME)

Visited locations and travel behaviour (ME)

Other factors: personality $(C$, $\mathrm{MO})$

Short Physical Performance Battery ${ }^{103}$ to assess lower-limb function and a dynamometer to measure hand grip

List of chronic diseases, health conditions and medications

Waist circumference, height and weight (assessed by staff)

Patient Health Questionnaire- $9^{48}$

\section{Soci} education, occupation, early life socioeconomic status, household composition) Jenkins Sleep Questionnaire ${ }^{104}$

Mediterranean Diet Adherence Screener questionnaire $^{105}$

Neuroticism and conscientiousness subscales of the NEO Five-Factor Inventory ${ }^{106}$

Reasons for living in the neighbourhood ${ }^{87}$

Smartphone geolocation data and travel diary data processed to extract information on locations and trips

Accelerometer data process to extract information on frequency, amount and intensity of physical activity

\section{Sedentary behaviour Lifestyle behaviours: \\ (frequency, duration, amount) sedentary behaviour (ME)} (O)

Route complexity (O)
Sleep quality (O, DL)

Lifestyle behaviour:
navigational activities (ME)
Lifestyle behaviours: sleep
(ME)
Accelerometer/inclinometer data

Smartphone geolocation data processed to derive an index of navigational complexity

Measured objectively using actigraphy ${ }^{107}$ and modified version of the Jenkins Sleep Questionnaire ${ }^{104}$

Daily positive and negative affective states (DL)

Mental health: Affective states IPANAS-SF included in daily $\log ^{108}$-time: today (ME)

Personal nitrogen dioxide $\left(\mathrm{NO}_{2}\right)$ Urban design by-products: air 7-day average personal $\mathrm{NO}_{2}$ exposure measured exposure $(\mathrm{O})$ pollution (E, ME) using monitors

Lifestyle behaviours: intellectual and social solving puzzles) and social activities (typical frequency, interview (VERITAS IMAP) activities (ME)
Visited locations (ME) duration, location, spatial and time constraints) (S)
Participants report socialising (in person, via the phone or internet) as an activity. Educational activities, certain work activities (managers, professional), reading, mental games and similar are classified as intellectual activities. 
Table 2 Continued

\section{Data collection \\ component \\ Constructs measured (type of assessment)}

Physical activity (typical frequency, duration, location, spatial and time constraints) (S)

\section{Construct in conceptual model (figure 1) (role in model)}

Lifestyle behaviours: physical activity (ME)

Visited locations and travel behaviour (ME)

Sedentary behaviour (typical Lifestyle behaviours: frequency, duration, location, sedentary behaviour (ME) spatial and time constraints) (S) Visited locations (ME)

Modes of transport to frequent Travel behaviour (ME) activity locations (S)

\begin{tabular}{|c|c|c|}
\hline & Perceived safety from crime (S) & Other factors: social (ME) \\
\hline & Home characteristics (S) & Visited locations (C, ME) \\
\hline & Social networks (S) & Other factors: social (ME) \\
\hline & $\begin{array}{l}\text { Length of residence at current } \\
\text { address and residential } \\
\text { addresses in last 10years (S) }\end{array}$ & $N A(C)$ \\
\hline \multirow{4}{*}{$\begin{array}{l}\text { GIS data and } \\
\text { environmental } \\
\text { audits }\end{array}$} & Dwelling density (GIS) & Urban design: density (E) \\
\hline & $\begin{array}{l}\text { Street intersection density } \\
\text { (GIS) }\end{array}$ & $\begin{array}{l}\text { Urban design: street network } \\
\text { connectivity (E) }\end{array}$ \\
\hline & Integration (GIS) & $\begin{array}{l}\text { Urban design: street network } \\
\text { connectivity }(E)\end{array}$ \\
\hline & Land use mix (GIS) & $\begin{array}{l}\text { Urban design: mixed land } \\
\text { use }(E)\end{array}$ \\
\hline
\end{tabular}

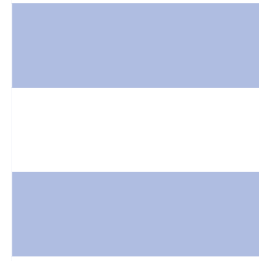

Destination availability and accessibility (GIS)

Urban design: diverse destinations and facilities (E)

Public transport availability and Urban design: transport accessibility (GIS) infrastructure $(E)$

Carpark availability and Urban design: transport accessibility (GIS)

Cycling lane availability and accessibility (GIS) infrastructure $(E)$

Urban design: cycling infrastructure $(E)$

Walking trail availability and accessibility (GIS)

Urban design: pedestrian infrastructure $(E)$

Slope (hilliness) (GIS)

Pavement (EA) infrastructure $(\mathrm{E})$

Urban design: pedestrian infrastructure $(E)$
Urban design: pedestrian

\section{Measure/assessment}

For sport and exercise activities, participants report the percentage of time spent in light, moderate and vigorous activities. For work related, household/ gardening and care-related activities participants report whether they involve mainly sitting, sitting and standing, walking with handling of light weights or walking and heavy manual work.

For work related, household/gardening and carerelated activities participants report whether they involve mainly sitting. Other types of activities are classified as sedentary or non-sedentary.

Usual modes of transport to a destination (multiple modes allowed); usual trip origin; travelling alone or with others

Four items from the Neighbourhood Environment Walkability Scale ${ }^{109}$

No of rooms, lighting, thermal comfort, maintenance, natural views, recreational areas

Size, relationship type, frequency of contact, interaction quality

Months/years at current address and location of previous residential addresses in last 10 years

Dwellings $/ \mathrm{km}^{2}$ within street network buffers

3-arm intersections $/ \mathrm{km}^{2}$ within street network buffers

Derived from the mean number of turns needed from a street segment to reach all other street segments in a buffer

Entropy index of 5 land uses (residential, commercial, industrial, institutional/civic and recreational) ${ }^{40}$

Street network distance to nearest destination of a specific type and number of destinations $/ \mathrm{km}^{2}$

Street network distance to nearest public transport stop and number of stops $/ \mathrm{km}^{2}$

Street network distance to nearest carpark and carparks $/ \mathrm{km}^{2}$

Street network distance to nearest cycling lane and length of cycling lane intersecting a street network buffer

Street network distance to nearest walking trail and length of walking trail intersecting a street network buffer

Average percentage slope in a street network buffer

Percentage of street segments with pavement along routes connecting residential/workplace addresses with the nearest commercial block assessed using MAPS Global ${ }^{50}$ 
Table 2 Continued

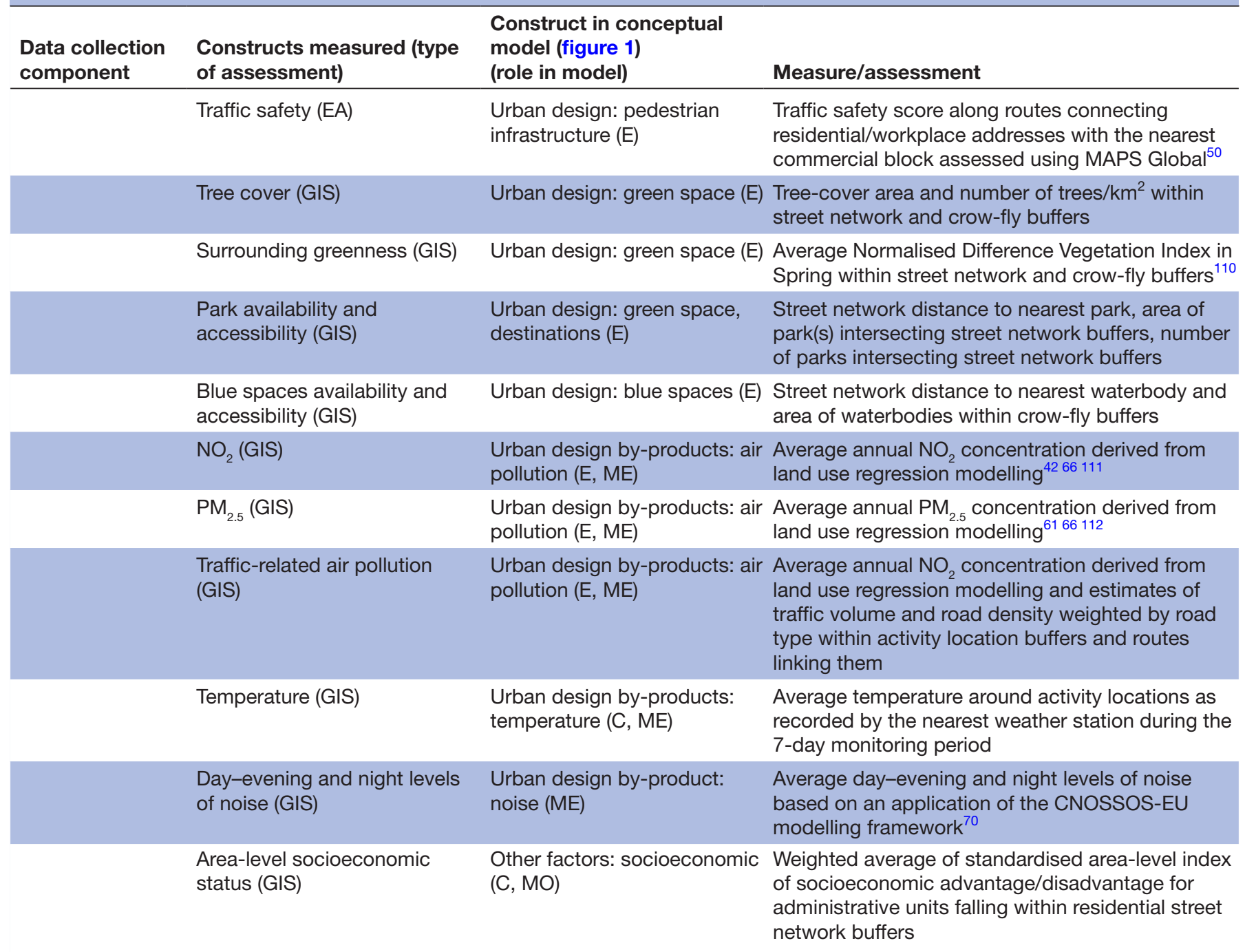

C, confounder; CNOSSOS, Common NOise aSSessment MethOdS in Europe; DL, daily log; E, exposure; EA, environmental audit; GIS, Geographic Information Systems; iMAP, International Mind, Activities and Urban Places; MAPS, Microscale Audit of Pedestrian Streetscapes; ME, mediator; MO, moderator; $\mathrm{NO}_{2}$, nitrogen dioxide; O, objective; S, self-report; TD, travel diary; VERITAS, Vizualization and Evaluation of Route Itineraries, Travel destinations and Activity Spaces.

for cognitive ill health, and they are widely measured by governments for regulatory purposes, supporting the development of exposure models. ${ }^{56-58}$ Pollution exposure can be categorised as general background ambient air pollution (air pollution from the range of sources including traffic, industry and natural, but not immediately influenced by nearby sources) and traffic-related air pollution in areas proximate to traffic (i.e., air pollution from cars, buses and trucks). Estimates of annual exposure to ambient air pollution for all participants at all assessment points will be computed. ${ }^{59}$ Nationwide satellitebased land use regression models for Australia (including Melbourne) have been developed and validated for $\mathrm{NO}_{2}$ and $\mathrm{PM}_{2,5}{ }^{42}{ }^{60-62}$ Air pollution surfaces for Barcelona have been developed within the European Study of Cohorts for Air Pollution Effects project, ${ }^{63}$ and those for Hong Kong by Barratt and colleagues. ${ }^{64-66}$ Traffic-related air pollution is operationalised as ambient $\mathrm{NO}_{2}$ as well as traffic volumes and road density weighted by road type ${ }^{67}$ within prespecified buffers around residences, workplaces and other visited locations. Participants are given the option of wearing a personal $\mathrm{NO}_{2}$ monitor (Gradko, UK) for seven consecutive days (as part of a 7-day field assessment) and completing a short survey regarding their pollution-related housing characteristics and typical activities. We focus on exposure to $\mathrm{NO}_{2}$ because it is considered an indicator of traffic-related air pollution. ${ }^{68}$ Additionally, $\mathrm{NO}_{2}$ (like other nitrogen oxides) is also an important contributor to the secondary formation of air pollutants, such as, ozone and particulate matter.

Annual day-evening (7:00 to 22:59) and night (23:00 to 6:59) levels of traffic-related noise exposures for all participants are estimated using a variant of the Common NOise aSSessment MethOdS in Europe noise modelling framework ${ }^{69}$ with relatively low-resolution inputs that are feasibly acquired across international study sites. ${ }^{70}$ The 
model uses information on traffic composition, flow and speed, road network land cover, building height, topography and meteorological conditions. The perceived acoustic environment at home (e.g., noise, sound pleasantness) is assessed using VERITAS iMAP. Information on the average temperature recorded by the weather stations nearest to locations visited by the participants during a 7-day activity monitoring period is collected. Finally, selfreport measures of neighbourhood safety from crime are assessed using VERITAS ${ }^{52}$ iMAP.

\section{Outcomes}

\section{Measures of cognitive function}

Cognitive function will be measured as part of the initial face-to-face assessment using reliable, standardised tests that have been previously used and validated in Australia, Spain and Hong Kong. These include paper-and-pencil versions of the:

1. $\mathrm{MoCA}^{47}$, a measure of global cognition used for cognitive impairment screening.

2. Symbol Digit Modality Test ${ }^{71}$ assessing psychomotor speed and attention.

3. Rey Auditory Verbal Learning Test ${ }^{72}$ assessing episodic memory.

4. Digit Span Backwards task ${ }^{73}$ measuring verbal working memory.

5. Victoria Stroop Test ${ }^{74}$ assessing the executive function of inhibitory control.

6. Colour Trail Test ${ }^{75}$ a measure of the executive function of switching.

7. Animal naming assessing semantic fluency-language. $^{76}$

8. A digital version of the Corsi Block Tapping Task, ${ }^{77} \mathrm{a}$ measure of visuospatial working memory.

The Symbol Digit Modality, Digit Span Backwards, Animal naming and Stroop tests have been shown to be sensitive to change over a 2-year period in non-demented adults aged 60 years and over, ${ }^{49} 78$ as have comparable tests of episodic memory, ${ }^{78-80}$ global cognition ${ }^{79} 80$ and visuospatial working memory. ${ }^{80}$

\section{Measures of brain health}

Brain health measures represent secondary outcomes as well as mediators of environment-cognitive health associations (figure 1). In a subsample of participants (300 in Melbourne, 200 in both Hong Kong and Barcelona), MRI scans are acquired at the local imaging labs using 3T MRI scanners. The MRI scans take approximately 45-50 mins and are focused on brain metrics related to neurodegeneration, brain ageing and oxidative stress and neuroplasticity (figure 1). The MRI protocol comprises T1-weighted three-dimensional (3D) MPRAGE (1 mm isotropic), T1-weighted high-resolution hippocampus $(0.4 \mathrm{~mm}$ coronal slices), 3D FLAIR $(1 \mathrm{~mm})$, diffusion weighted imaging (DWI; b-value $=1000$ ), and T $2 *$-weighted gradient-echo (GRE)/susceptibly weighted imaging (SWI) sequences. The 3D MPRAGE and hippocampus MRI data are processed to quantify cortical thickness, surface area and grey matter density of brain regions, volume of the hippocampus and its subfields, and ventricular volumes (neurodegeneration; figure 1). ${ }^{81} 82$ The 3D FLAIR MRI is administered to obtain volume and number of white matter hyperintensities (an index of white matter injury) associated with neuroinflammation and vascular changes in the brain (brain ageing and oxidative stress; figure 1) ${ }^{83}$ The DWI data are used to measure fractional anisotropy and diffusivity values of white matter tracts and regions (e.g., cingulum bundle and fornix), allowing us to probe any microstructural changes associated with neuroplasticity. ${ }^{84}$ The GRE/SWI data are used to identify the number of microbleeds and estimate susceptibility changes due to iron loading associated with ageing and progressive neurodegeneration. ${ }^{85}$ The MRI protocols are harmonised across study sites by following current recommendations for multi-site MRI study protocols. ${ }^{86}$

\section{Covariates, moderators and/or mediators}

Tools used to collect data on visited locations, travel behaviour, lifestyle behaviours, mental health, cardiometabolic health and 'other factors' included in the ecological model of the effects of urban design on brain and cognitive health (figure 1) are reported in table 2 by data collection component. Most of these variables will be treated as potential covariates or mediators of the associations between environmental characteristics and brain and cognitive health. ApoE genotype, area-level and individual-level SES indicators, personality traits, chronic health conditions (e.g., diabetes) and social environment variables (e.g., perceived safety from crime) will also be examined as moderators of associations (figure 1). Virtually all the measures proposed have been evaluated for reliability and validity, and most have been widely used. This study also collects data on length of residence at the current address and other residential addresses in the 10 years prior to more accurately quantify long-term environmental exposures. In addition, reasons for moving to (or living in) the current neighbourhood ${ }^{87}$ are recorded to help address residential self-selection bias (individuals self-selecting into their residential environment based on previously determined preferences for travel or engagement in activities). ${ }^{88}$

The 7-day field assessment collects data on lifestyle activities and their locations, travel behaviour and affective states in the participants' habitual environments for seven consecutive days. Participants wear a wrist-worn monitor and thigh-attached inclinometer for 24 hours/ day and a waist-mounted matchbox-sized accelerometer, a smartphone and (optionally) a personal $\mathrm{NO}_{2}$ monitor during waking hours ( $\geq 10$ hours/day). To objectively assess visited locations, modes of transport and trip routes over 7 days, smartphone geolocation data are used. Waistworn accelerometer data are used to derive average minutes/day of light, moderate and vigorous physical activity, while the inclinometer is used to assess sedentary behaviour using established protocols. ${ }^{89} 90$ We quantify complexity of trip routes (i.e., the cognitive difficulty of 
navigational activities) in terms of length, street intersections crossed and number of turns at intersections using GIS software. Wrist-worn accelerometer data are used to quantify total sleep time and efficiency, waking after sleep onset and number of long wake episodes. Participants keep a paper-and-pencil travel diary where they report the time of a trip, destination and modes of transport. At the start of each day, participants complete a short sleep quality questionnaire, while at the end of each day, participants complete a short questionnaire on affective states (table 2).

Apart from collecting information on habitually visited locations and their characteristics, VERITAS iMAP is used to assess types, frequency and duration of intellectual, social, physical activities and sedentary behaviours (as appropriate) for each reported habitual location; the extent to which it is difficult to change the timing and location of the activity reported within a specific location; with whom they do the activities; and transport modes to visited locations. The participants are first asked if they usually visit a specific type of location (e.g., shopping mall) and, using map search capabilities, the locations are found on the map and confirmed by the participants. Then, geographical coordinates, names of destinations and additional information (e.g., frequency of visits and mode of transport to get to/from the destination, types and frequency of activities undertaken) are recorded in a spatial database.

\section{Data collection and procedures}

At each study wave, data collection on all participants include:

1. A face-to-face sociodemographic, health and cognitive function assessment.

2. A self-completed survey.

3. A 7-day field assessment of lifestyle behaviours and their locations, travel behaviour, affective states and, optionally, personal $\mathrm{NO}_{2}$ exposure using passive samplers.

4. A semistructured map-assisted interview about habitually visited locations, lifestyle activities, travel behaviour and social networks.

5. Objective assessment of the environment using both GIS and environmental audit data.

Subsamples of 200 (Barcelona and Hong Kong) and 300 (Melbourne) participants are invited to undertake MRI brain scans within 10 weeks after the completion of the other assessments. Data collection is being conducted in 2019/2021 and repeated 24 months later in 2021/2023.

After the provision of written consent and rescreening for eligibility, eligible participants undergo a series of health and cognitive assessments and are given a selfadministered paper-and-pencil survey to complete in their own time as detailed in table 2. They are also provided with the material and instructions for the 7-day field assessment of lifestyle activities described above. At the end of the field assessment, participants undertake a face-to-face semistructured map-assisted interview about their frequently visited locations, lifestyle activities, travel behaviour and social networks (VERITAS iMAP). Finally, eligible and consenting participants are randomly selected for MRI brain scans from the eight types of neighbourhoods in a balanced manner. Objective assessment of the participants' environment entails linking the geocoded locations participants regularly visit (according to data from VERITAS iMAP) and visited during the 7-day field assessment period (plus routes between them) to objective GIS and environmental-audit data of interest (e.g., dwelling density or distance to a park). Various strategies for participants' retention are employed. These include the provision of short individualised reports on lifestyle behaviours, motivational phone calls, brief newsletters about the study progress and birthday cards.

\section{Data analyses}

Associations of environmental attributes with levels and 24 months changes in cognitive function, lifestyle behaviours and brain health will be estimated using Generalised Additive Mixed Models (GAMMs), which are able to detect curvilinear relationships and account for administrative unit-level and person-level (repeated measures) clustering, and appropriate for data with different distributions. ${ }^{91}$ Eight outcome measures of cognitive function will be examined. These include seven domain-specific cognitive function tests (listed above) and a composite measure of executive function based on four of these tests (Colour Trail Test, Victoria Stroop Test, Digit Span Backwards task and the Corsi Block Tapping Task). City will be included as a covariate in the GAMMs and also as a moderator to examine whether the observed effects vary by city. Directed Acyclic Graphs will be employed to select confounders to be included in the models. Moderating effects will be examined by adding appropriate interaction terms to the main effect GAMMs. Mediation analyses will be conducted using traditional (e.g., the product-of-coefficients method or joint-significance test with AU cluster bootstrapped confidence intervals ${ }^{92}$ and state-of-the-art causal inference approaches, ${ }^{93}$ as appropriate, while paying attention to model validity. To avoid collinearity, composite measures of collinear variables will be included in multiple-predictor models, while also assessing whether a specific component of the composite measure is associated with the outcome. No correction for multiple testing will be adopted as this work is confirmatory and hypothesis based. Missing data will be addressed by multiple imputation techniques following best-practice procedures to minimise sources of bias.

Particular attention will be paid to strengthen the evidence of causality. To address the possibility of reverse causality (cognitive functioning determining the place of residence and activities), only cognitively intact older adults will be recruited. Reasons for living in the (current) neighbourhood (e.g., health issues) and information on residential addresses in the last 10 years will be available and incorporated in the models. To address the issue of lost to follow-up associated with cognitive impairment, 
we will conduct sensitivity analyses. First, GAMMs will produce reliable estimates under the assumption of data missing at random. Then, we will use multilevel logistic regression to fit predictive models of drop-out at the second assessment and compute weights representing the inverse probability of attrition for each participant. ${ }^{94}$ These weights will be used in weighted GAMMs to account for drop-outs associated with cognitive impairment (data 'not missing at random'). We will contrast the regression estimates from the two sets of models to quantify the bias due to selective attrition.

\section{Patient and public involvement}

This study does not target participants with specific health conditions (patients). Participants are independently living community dwellers who receive individually tailored reports on a set of lifestyle behaviours (e.g., weekly sedentary time and physical activity) after each data collection wave. Members of the public were not involved in the design of the study or recruitment. However, they will be involved in the dissemination of findings, which will be disseminated to the wider public via the local iMAP websites, media and non-government and local community organ

\section{ETHICS AND DISSEMINATION}

On recruitment, participants are given detailed verbal and written information on the study and, after their eligibility is assessed, they are requested to provide written informed consent before the start of the first face-to-face assessment. Only the named researchers have access to the data that will be digitally stored for 20 years in a secured environment and permanently destroyed thereafter. All data are kept private and confidential.

At the completion of first and second data collection waves, results will be provided to key stakeholders and organisations (e.g., public health professionals, national and provincial urban planning associations, clinicians and participants). Dissemination of study methods and findings will be designed to impact both science and policy. The methods and measures used will be made freely available, and training will be provided as needed, to help these methods become a worldwide standard. Anonymised, aggregate results will be communicated to the scientific community through publications in high-impact journals and presentations in diverse international conferences. A publication committee will encourage and coordinate all papers, from initial proposal and establishment of an international writing team, to analysis plans, through quality control of manuscripts. Results will be communicated to practitioners and policy makers, targeting especially health, urban design/planning, housing and transport sectors. Delivery modes will be press releases, policy briefs, a website (https://ichen.site/imap/), webinars, presentations at conferences, and consultations with national and international advocacy organisations.

\section{DISCUSSION}

iMAP is an international data-rich, comprehensive project aiming to examine how urban environments influence brain and cognitive health in mid-aged and older community dwellers. By recruiting participants from cities and neighbourhoods with diverse geographical and sociocultural characteristics, this study will enable expansion of variability in exposures and, hence, allow a robust estimation of dose-response environment-behaviour-cognitive health relationships. International studies on urban environments and behaviours (physical activity) using common objective and self-report measures like those employed in iMAP have demonstrated the utility, feasibility and superior statistical power of this type of design. These studies yielded findings that were generalisable across a dozen of countries from five continents ${ }^{37} 3895$ indicating that a carefully developed data collection protocol and ongoing quality checks can minimise differences between study sites due to methodological artefacts and, thus, allow meaningful data pooling. ${ }^{46}$ However, in the presence of differences in effects across sites, it is often difficult to ascertain the factors causing such differences. Yet, this argument also applies to data from single-site studies as participants from different ethnic backgrounds may respond to test items in different ways. ${ }^{96}$

There are some limitations to iMAP. In Barcelona and, when necessary, in Melbourne, participants will be/ are being recruited via street intercepts in preselected administrative units. This type of recruitment can result in a biased sample. Another limitation pertains to the number of assessments and the interval between them. iMAP has secured funding to collect two waves of data (2 years apart) on environmental characteristics, lifestyle behaviours and brain and cognitive health. As 2 years may be insufficient to detect changes in certain exposures and/or outcomes (a study limitation), additional funds will be sought to support two more waves of data collection, extending the monitoring period to 6 years. In this regard, the multisite multiethnic study on atherosclerosis has reported changes in environmental attributes across a 5-year period ranging from $0 \%$ to $53 \%$ (2-year change: $0 \%-20 \%$ ), which varied across study sites and were generally smaller for population density and residential land use and larger for access to amenities. ${ }^{97}$ This indicates that the data from the first two waves may not have enough power to detect the effects of changes in some environmental attributes on the outcomes. With regard to brain health, a previous study of 127 community dwellers was powered to detect associations between environmental attributes and 1.5-year changes in brain volume. ${ }^{21}$ However, the participants were on average 10 years older than the iMAP sample. Also, significant 1.5year changes on 6 out of 12 cognitive function measures were detected in 685 cognitively intact participants with a mean age of 67 years (projected average age in iMAP: 65 years). ${ }^{49}$ These included a measure of global cognition and versions of the Stroop and digit span tests, which are part of the iMAP cognitive assessment. 
Author affiliations

${ }^{1}$ Mary MacKillop Inst Health Res, Australian Catholic University, Melbourne, Victoria, Australia

${ }^{2}$ School of Public Health, University of Hong Kong, Hong Kong, Hong Kong SAR,

China

${ }^{3}$ INSERM, Institut Pierre Louis d'Épidémiologie et de Santé Publique, Sorbonne Université, Paris, Île-de-France, France

${ }^{4}$ Instituto de Salud Global Barcelona, Barcelona, Catalunya, Spain

${ }^{5}$ Cognitive Neurosciences Unit, Deakin University, Burwood, Victoria, Australia

${ }^{6}$ Population Health Intelligence, Healthy People and Places Unit, South Western Sydney Local Health District, Sydney, New South Wales, Australia

${ }^{7}$ Centre for Urban Transitions, Swinburne University of Technology, Hawthorn, Victoria, Australia

${ }^{8}$ Department of Family Medicine and Public Health, University of California San Diego, La Jolla, California, USA

${ }^{9}$ Department of Psychiatry, The University of Melbourne, Melbourne, Victoria, Australia

${ }^{10}$ School of Public Health, The University of Queensland, Herston, Queensland, Australia

${ }^{11}$ Baker Heart and Diabetes Institute, Melbourne, Victoria, Australia

${ }^{12}$ UNSW Ageing Futures Institute and School of Psychology, University of New South

Wales, Sydney, New South Wales, Australia

${ }^{13}$ Neuroscience Research Australia, Randwick, New South Wales, Australia

Acknowledgements We are grateful for the contributions of Erika Martino and David H Lee (selection of administrative units in Melbourne), Marta Cirach (selection of administrative units in Barcelona), Casper JP Zhang (selection of administrative units in Hong Kong), Karen Biddiscombe (selection of cognitive measures), Sonia Marchionda (selection of dietary measures) and Nerolie Stickland (development of 7-day assessment protocols).

Contributors EC wrote and AB, MJN, KC, NL, BJ, BC, TS, DWD, KJA, LT and JFS contributed to the original grant proposal on which the study design and paper is based. GP, AJW, RT, Y-KC, MYN, AC and DD-G contributed to modifications of the original grant proposal. LK modelled air pollution estimates for the selection of administrative units in Melbourne. $\mathrm{EC}$ and $\mathrm{AB}$ drafted this version of the paper and received input from all the authors. All authors read and commented on the paper and agreed with the final version.

Funding The study is part of a programme grant primarily funded by an Australian Catholic University Research Fund (ACURF-2018). The establishment of the FAMILY cohort was funded by the Hong Kong Jockey Club Charities Trust from 2007 to 2014.

Competing interests None declared.

Patient consent for publication Not required.

Ethics approval The study protocol has been approved by the Human Research Ethics Committee of the Australian Catholic University (2018-191 H), the Institutional Review Board of the University of Hong Kong (UW 19-126) and the Parc de Salut Mar Clinical Research Ethics Committee of the Government of Catalonia (2019/8469/l).

\section{Provenance and peer review Not commissioned; externally peer reviewed.}

Open access This is an open access article distributed in accordance with the Creative Commons Attribution Non Commercial (CC BY-NC 4.0) license, which permits others to distribute, remix, adapt, build upon this work non-commercially, and license their derivative works on different terms, provided the original work is properly cited, appropriate credit is given, any changes made indicated, and the use is non-commercial. See: http://creativecommons.org/licenses/by-nc/4.0/.

ORCID iD

Ester Cerin http://orcid.org/0000-0002-7599-165X

\section{REFERENCES}

1 Prince M, Albanese E, Guerchet M, et al. World Alzheimer report 2014: dementia and risk reduction: an analysis of protective and modifiable risk factors. London: Alzheimer's Disease International, 2014.

2 GBD. Dementia Collaborators. Global, regional, and national burden of Alzheimer's disease and other dementias, 1990-2016: a systematic analysis for the Global Burden of Disease Study 2016. Lancet Neurol 2016;2019:88-106.

3 Australian Institute of Health and Welfare. Dementia in Australia. Canberra: Australian Government, 2012.

4 Lin P-J, Neumann PJ. The economics of mild cognitive impairment. Alzheimers Dement 2013;9:58-62.

5 Sachdev PS, Lipnicki DM, Kochan NA, et al. The prevalence of mild cognitive impairment in diverse geographical and ethnocultural regions: the COSMIC collaboration. PLoS One 2015;10:e0142388.

6 Montine TJ, Cholerton BA, Corrada MM, et al. Concepts for brain aging: resistance, resilience, reserve, and compensation. Alzheimers Res Ther 2019;11:22.

7 Stern Y. Cognitive reserve. Neuropsychologia 2009;47:2015-28.

8 IOM. Institute of medicine). cognitive aging: progress in understanding and opportunities for action. Washington, DC: The National Academies Press, 2015.

9 Stokols D. Translating social ecological theory into guidelines for community health promotion. Am J Health Promot 1996;10:282-98.

10 United Nations, Department of Economic and Social Affairs, Population Division. World urbanizations prospects: 2014 revision. Geneva UN; 2014. https://esa.un.org/indp/wup/Publications/Files/ WUP2014-Highlights.pdf

11 Moore M, Gould P, Keary BS. Global urbanization and impact on health. Int J Hyg Environ Health 2003;206:269-78.

12 Nieuwenhuijsen MJ. Urban and transport planning, environmental exposures and health-new concepts, methods and tools to improve health in cities. Environ Health 2016;15 Suppl 1:38.

13 Stevenson M, Thompson J, de Sá TH, et al. Land use, transport, and population health: estimating the health benefits of compact cities. Lancet 2016;388:2925-35.

14 Cerin E, Nathan A, van Cauwenberg J, et al. The neighbourhood physical environment and active travel in older adults: a systematic review and meta-analysis. Int J Behav Nutr Phys Act 2017;14:15.

15 Barnett DW, Barnett A, Nathan A, et al. Built environmental correlates of older adults' total physical activity and walking: a systematic review and meta-analysis. Int J Behav Nutr Phys Act 2017; $14: 103$.

16 Van Cauwenberg J, Nathan A, Barnett A, et al. Relationships between neighbourhood physical environmental attributes and older adults' leisure-time physical activity: a systematic review and meta-analysis. Sports Med 2018;48:1635-60.

17 Leal C, Chaix B. The influence of geographic life environments on cardiometabolic risk factors: a systematic review, a methodological assessment and a research agenda. Obes Rev 2011;12:217-30.

18 Sallis JF, Floyd MF, Rodríguez DA, et al. Role of built environments in physical activity, obesity, and cardiovascular disease. Circulation 2012;125:729-37.

19 Besser LM, McDonald NC, Song Y, et al. Neighborhood environment and cognition in older adults: a systematic review. $\mathrm{Am}$ $J$ Prev Med 2017;53:241-51.

$20 \mathrm{Ng}$ TP, Nyunt MSZ, Shuvo FK, et al. The neighborhood built environment and cognitive function of older persons: results from the Singapore longitudinal ageing study. Gerontology 2018:64:149-56.

21 Cerin E, Rainey-Smith SR, Ames D, et al. Associations of neighborhood environment with brain imaging outcomes in the Australian imaging, biomarkers and lifestyle cohort. Alzheimers Dement 2017;13:388-98.

22 Besser LM, Rodriguez DA, McDonald N, et al. Neighborhood built environment and cognition in non-demented older adults: the multi-ethnic study of atherosclerosis. Soc Sci Med 2018;200:27-35.

23 Wu Y-T, Prina AM, Jones A, et al. The built environment and cognitive disorders: results from the cognitive function and ageing study II. Am J Prev Med 2017;53:25-32.

24 Cerin E. Building the evidence for an ecological model of cognitive health. Health Place 2019;60:102206.

25 Jackson PA, Pialoux V, Corbett D, et al. Promoting brain health through exercise and diet in older adults: a physiological perspective. J Physiol 2016;594:4485-98.

26 Blackwell T, Yaffe K, Laffan A, et al. Associations of objectively and subjectively measured sleep quality with subsequent cognitive decline in older community-dwelling men: the MROS sleep study. Sleep 2014;37:655-63.

27 Chen $\mathrm{H}$, Kwong JC, Copes R, et al. Living near major roads and the incidence of dementia, Parkinson's disease, and multiple sclerosis: a population-based cohort study. Lancet 2017;389:718-26.

28 Feigin VL, Roth GA, Naghavi M, et al. Global burden of stroke and risk factors in 188 countries, during 1990-2013: a systematic analysis for the global burden of disease study 2013. Lancet Neurol 2016;15:913-24. 
29 Health Effects Institute. Traffic-Related air pollution: a critical review of the literature on emissions, exposure, and health effects. Special report; 2010. http://pubs.healtheffects.org/getfile.php?u= 553

30 Brown BM, Peiffer JJ, Taddei K, et al. Physical activity and amyloid- $\beta$ plasma and brain levels: results from the Australian imaging, biomarkers and lifestyle study of ageing. Mol Psychiatry 2013;18:875-81.

31 Lee BK, Glass TA, James BD, et al. Neighborhood psychosocial environment, apolipoprotein $\mathrm{E}$ genotype, and cognitive function in older adults. Arch Gen Psychiatry 2011;68:314-21.

32 Cacciottolo M, Wang X, Driscoll I, et al. Particulate air pollutants, APOE alleles and their contributions to cognitive impairment in older women and to amyloidogenesis in experimental models. Transl Psychiatry 2017;7:e1022.

33 Mangold DL, Wand GS. Cortisol and adrenocorticotropic hormone responses to naloxone in subjects with high and low neuroticism. Biol Psychiatry 2006;60:850-5.

34 Eysenck HJ, Personality EMW. Individual differences: a natural science approach. New York: Plenum, 1985.

35 Lebedeva A, Sundström A, Lindgren L, et al. Longitudinal relationships among depressive symptoms, cortisol, and brain atrophy in the neocortex and the hippocampus. Acta Psychiatr Scand 2018;137:491-502.

36 Vandenbroucke JP, von Elm E, Altman DG, et al. Strengthening the reporting of observational studies in epidemiology (STROBE): explanation and elaboration. PLoS Med 2007;4:e297.

37 Cerin E, Cain KL, Conway TL, et al. Neighborhood environments and objectively measured physical activity in 11 countries. Med Sci Sports Exerc 2014;46:2253-64.

38 Sallis JF, Cerin E, Conway TL, et al. Physical activity in relation to urban environments in 14 cities worldwide: a cross-sectional study. Lancet 2016;387:2207-17.

39 Cerin E, Sit CHP, Zhang CJP, et al. Neighbourhood environment, physical activity, quality of life and depressive symptoms in Hong Kong older adults: a protocol for an observational study. BMJ Open 2016;6:e010384.

40 Frank L, Pivo G. Impacts of mixed use and density on utilization of three modes of travel: single-occupant vehicle, transit, and walking Transport Res Rec 1994;1466:44-52.

41 WHO Regional Office for Europe. Review of evidence on health aspects of air pollution - REVIHAAP Project: Technical Report [Internet]. Copenhagen: WHO Regional Office for Europe 2013. C Proximity to roads, NO2, other air pollutants and their mixtures. Available from:https://www.ncbi.nlm.nih.gov/books/NBK361807/

42 Knibbs LD, Hewson MG, Bechle MJ, et al. A national satellite-based land-use regression model for air pollution exposure assessment in Australia. Environ Res 2014;135:204-11.

43 Snijders T, Bosker R. Multilevel analysis: an introduction to basic and advanced multilevel modelling. London, UK: Sage Publications, 1999.

44 Kerr J, Sallis JF, Owen N, et al. Advancing science and policy through a coordinated international study of physical activity and built environments: IPEN adult methods. J Phys Act Health 2013;10:581-601.

45 Leung GM, Ni MY, Wong PT, et al. Cohort profile: family cohort. Int J Epidemiol 2017;46:e1. 1.

46 Sachdev PS, Brodaty H, Reppermund S, et al. The Sydney memory and ageing study (MAS): methodology and baseline medical and neuropsychiatric characteristics of an elderly epidemiological nondemented cohort of Australians aged 70-90 years. Int Psychogeriatr 2010;22:1248-64.

47 Nasreddine ZS, Phillips NA, Bédirian V, et al. The Montreal cognitive assessment, MoCA: a brief screening tool for mild cognitive impairment. J Am Geriatr Soc 2005;53:695-9.

48 Kroenke K, Spitzer RL, Williams JB. The PHQ-9: validity of a brief depression severity measure. J Gen Intern Med 2001;16:606-13.

49 Ellis KA, Szoeke C, Bush Al, et al. Rates of diagnostic transition and cognitive change at 18-month follow-up among 1,112 participants in the Australian imaging, biomarkers and lifestyle Flagship study of ageing (AIBL). Int Psychogeriatr 2014;26:543-54.

50 Cain KL, Geremia CM, Conway TL, et al. Development and reliability of a streetscape observation instrument for international use: MAPS-global. Int J Behav Nutr Phys Act 2018;15:19.

51 Phillips CB, Engelberg JK, Geremia CM, et al. Online versus inperson comparison of microscale audit of pedestrian Streetscapes (maps) assessments: reliability of alternate methods. Int $\mathrm{J}$ Health Geogr 2017;16:27.

52 Chaix B, Kestens Y, Perchoux C, et al. An interactive mapping tool to assess individual mobility patterns in neighborhood studies. Am J Prev Med 2012;43:440-50.
53 Clougherty JE, Wright RJ, Baxter LK, et al. Land use regression modeling of intra-urban residential variability in multiple trafficrelated air pollutants. Environ Health 2008;7:17.

54 Kwan M-P. Space-Time and integral measures of individual accessibility: a comparative analysis using a point-based framework. Geogr Anal 1998;30:191-216.

55 Christiansen LB, Cerin E, Badland H, et al. International comparisons of the associations between objective measures of the built environment and transport-related walking and cycling: IPEN adult study. J Transp Health 2016;3:467-78.

56 Heusinkveld $\mathrm{HJ}$, Wahle T, Campbell A, et al. Neurodegenerative and neurological disorders by small inhaled particles. Neurotoxicology 2016;56:94-106.

57 Killin LOJ, Starr JM, Shiue IJ, et al. Environmental risk factors for dementia: a systematic review. BMC Geriatr 2016;16:175.

58 Peters R, Peters J, Booth A, et al. Is air pollution associated with increased risk of cognitive decline? A systematic review. Age Ageing 2015;44:755-60.

59 Hoek G, Beelen R, de Hoogh K, et al. A review of land-use regression models to assess spatial variation of outdoor air pollution. Atmos Environ 2008;42:7561-78.

60 Knibbs LD, Coorey CP, Bechle MJ, et al. Independent validation of national satellite-based land-use regression models for nitrogen dioxide using passive samplers. Environ Sci Technol 2016;50:12331-8.

61 Knibbs LD, van Donkelaar A, Martin RV, et al. Satellite-Based Land-Use Regression for Continental-Scale Long-Term Ambient $\mathrm{PM}_{25}$ Exposure Assessment in Australia. Environ Sci Technol 2018;52:12445-55.

62 Pereira G, Lee HJ, Bell M, et al. Development of a model for particulate matter pollution in Australia with implications for other satellite-based models. Environ Res 2017;159:9-15.

63 Beelen R, Raaschou-Nielsen O, Stafoggia M, et al. Effects of long-term exposure to air pollution on natural-cause mortality: an analysis of 22 European cohorts within the multicentre escape project. Lancet 2014;383:785-95.

64 Wong PPY, Lai P-C, Allen R, et al. Vertical monitoring of trafficrelated air pollution (TRAP) in urban street canyons of Hong Kong. Sci Total Environ 2019;670:696-703.

65 Tang R, Tian L, Thach T-Q, et al. Integrating travel behavior with land use regression to estimate dynamic air pollution exposure in Hong Kong. Environ Int 2018;113:100-8.

66 Lee M, Brauer M, Wong P, et al. Land use regression modelling of air pollution in high density high rise cities: a case study in Hong Kong. Sci Total Environ 2017;592:306-15.

67 Rose N, Cowie C, Gillett R, et al. Weighted road density: a simple way of assigning traffic-related air pollution exposure. Atmos Environ 2009;43:5009-14.

68 European Environment Agency. Air quality in Europe - 2016 report No. 28/2016. Luxembourg European Environment Agency; 2016.

69 Kephalopoulos S, Paviotti M, Anfosso-Ledee F. Common noise assessment methods in Europe (CNOSSOS-EU) Eur 25379 en. publications office. Luxemburg: of the European Union, 2012.

70 Morley DW, de Hoogh K, Fecht D, et al. International scale implementation of the CNOSSOS-EU road traffic noise prediction model for epidemiological studies. Environ Pollut 2015;206:332-41.

71 Smith A. Symbol digit modalities test: manual. Los Angeles, CA: WPS, 1998

72 Rey A. L'Examen Clinique en Psychologie. Paris: Presses Universitaries de France, 1964

73 Strauss E, Sherman EMS, Spreen O. Compendium of neuropsychological tests: administration, norms, and commentary. (3rd ed.). New York: Oxford University Press, 2006.

74 Spreen O. Strauss EA compendium of neuropsychological tests. 2nd ed. New York: Oxford University Press, 1998..

75 D'Elia LF, Satz P, Uchiyama CL, et al. Color trails test. Odessa, FL: PAR, 1996.

76 Spreen O, Benton AL. Neurosensory centre comprehensive examination for aphasia manual NCCEA. Victoria: University of Victoria, 1969

77 Vandierendonck A, Kemps E, Fastame MC, et al. Working memory components of the Corsi blocks task. Br J Psychol 2004;95:57-79.

78 Zaninotto P, Batty GD, Allerhand M, et al. Cognitive function trajectories and their determinants in older people: 8 years of follow-up in the English longitudinal study of ageing. J Epidemiol Community Health 2018;72:685-94.

79 Li LW, Ding D, Wu B, et al. Change of cognitive function in U.S. Chinese older adults: a population-based study. J Gerontol A Biol Sci Med Sci 2017;72:S5-10. suppl_1. 
80 Okonkwo OC, Cohen RA, Gunstad J, et al. Longitudinal trajectories of cognitive decline among older adults with cardiovascular disease. Cerebrovasc Dis 2010;30:362-73.

81 Poudel GR, Harding IH, Egan GF, et al. Network spread determines severity of degeneration and disconnection in Huntington's disease. Hum Brain Mapp 2019;40:4192-201.

82 Acosta O, Bourgeat P, Zuluaga MA, et al. Automated voxel-based $3 \mathrm{D}$ cortical thickness measurement in a combined LagrangianEulerian PDE approach using partial volume maps. Med Image Anal 2009;13:730-43.

83 Griffanti L, Zamboni G, Khan A, et al. BIANCA (brain intensity abnormality classification algorithm): a new tool for automated segmentation of white matter hyperintensities. Neuroimage 2016;141:191-205.

84 Caeyenberghs $\mathrm{K}$, Clemente A, Imms P, et al. Evidence for trainingdependent structural neuroplasticity in brain-injured patients: a critical review. Neurorehabil Neural Repair 2018;32:99-114.

85 Domínguez JFD, Ng ACL, Poudel G, et al. Iron accumulation in the basal ganglia in Huntington's disease: cross-sectional data from the IMAGE-HD study. J Neurol Neurosurg Psychiatry 2016;87:545-9.

86 Zavaliangos-Petropulu A, Nir TM, Thomopoulos SI, et al. Diffusion $\mathrm{MRI}$ indices and their relation to cognitive impairment in brain aging: the updated multi-protocol approach in ADNI3. Front Neuroinform 2019;13:2.

87 Cerin E, Leslie E, du Toit L, et al. Destinations that matter: associations with walking for transport. Health Place 2007;13:713-24.

88 Cao X, Mokhtarian PL, Handy SLVenezia E, ed. Examining the impacts of residential self-selection on travel behavior: methodologies and empirical findings; in urban sustainable mobility Milan: FrancoAngeli, 2011: 15-100.

89 Barnett $A$, van den Hoek D, Barnett $D$, et al. Measuring moderateintensity walking in older adults using the ActiGraph accelerometer. BMC Geriatr 2016;16:211.

90 Edwardson CL, Winkler EAH, Bodicoat DH, et al. Considerations when using the activPAL monitor in field-based research with adult populations. J Sport Health Sci 2017;6:162-78.

91 Wood SN. Generalized additive models: an introduction with $R$. Boca Raton: Chapman and Hall, 2006.

92 Cerin E. Ways of unraveling how and why physical activity influences mental health through statistical mediation analyses. Ment Health Phys Act 2010;3:51-60.

93 VanderWeele TJ. Mediation analysis: a practitioner's guide. Annu Rev Public Health 2016;37:17-32.

94 Weuve J, Tchetgen Tchetgen EJ, Glymour MM, et al. Accounting for bias due to selective attrition: the example of smoking and cognitive decline. Epidemiology 2012;23:119-28.

95 Kerr J, Emond JA, Badland $\mathrm{H}$, et al. Perceived neighborhood environmental attributes associated with walking and cycling for transport among adult residents of 17 cities in 12 countries: the IPEN study. Environ Health Perspect 2016;124:290-8.
96 Filshtein T, Chan M, Mungas D, et al. Differential item functioning of the everyday cognition (ECoG) scales in relation to racial/ethnic groups. J Int Neuropsychol Soc 2020;24:1-12.

97 Hirsch JA, Moore KA, Clarke PJ, et al. Changes in the built environment and changes in the amount of walking over time: longitudinal results from the multi-ethnic study of atherosclerosis. Am J Epidemiol 2014:180:799-809.

98 Australian Bureau of Statistics. National health survey: first results, 2014-15. Canberra: ABS; 2016.

99 Pardo A, Román-Viñas B, Ribas-Barba L, et al. Health-Enhancing physical activity and associated factors in a Spanish population. $J$ Sci Med Sport 2014;17:188-94.

100 Bauman A, Bull F, Chey T, et al. The International prevalence study on physical activity: results from 20 countries. Int J Behav Nutr Phys Act 2009;6:21.

101 Wu Y-T, Lee H-Y, Norton S, et al. Prevalence studies of dementia in mainland China, Hong Kong and Taiwan: a systematic review and meta-analysis. PLoS One 2013;8:e66252.

102 Perera G, Pedersen L, Ansel D, et al. Dementia prevalence and incidence in a Federation of European electronic health record databases: the European medical informatics framework resource. Alzheimers Dement 2018;14:130-9.

103 Guralnik JM, Simonsick EM, Ferrucci L, et al. A short physical performance battery assessing lower extremity function: association with self-reported disability and prediction of mortality and nursing home admission. J Gerontol 1994:49:M85-94.

104 Jenkins CD, Stanton BA, Niemcryk SJ, et al. A scale for the estimation of sleep problems in clinical research. $J$ Clin Epidemiol 1988:41:313-21.

105 Martínez-González MA, García-Arellano A, Toledo E, et al. A 14-item Mediterranean diet assessment tool and obesity indexes among high-risk subjects: the PREDIMED trial. PLoS One 2012;7:e43134

106 Costa PT, McCrae RR. Neo PI-R professional manual. revised Neo personality inventory (Neo Pl-R) and Neo Five-Factor inventory (NEO-FFI. Odessa: Psychological Assessment Resources, 1992.

107 Martin JL, Hakim AD. Wrist actigraphy. Chest 2011;139:1514-27.

108 Thompson ER. Development and validation of an internationally reliable short-form of the positive and negative affect schedule (PANAS). J Cross Cult Psychol 2007;38:227-42.

109 Cerin E, Conway TL, Cain KL, et al. Sharing good news across the world: developing comparable scores across 12 countries for the neighborhood environment Walkability scale (news). BMC Public Health 2013;13:309.

110 Tucker CJ. Red and photographic infrared linear combinations for monitoring vegetation. Remote Sens Environ 1979;8:127-50.

111 Beelen R, Hoek G, Vienneau D, et al. Development of NO2 and Nox land use regression models for estimating air pollution exposure in 36 study areas in Europe - the escape project. Atmos Environ 2013;72:10-23.

112 Eeftens M, Beelen R, de Hoogh K, et al. Development of Land Use Regression models for PM(2.5), PM(2.5) absorbance, PM(10) and PM(coarse) in 20 European study areas; results of the ESCAPE project. Environ Sci Technol 2012;46:11195-205. 